\title{
Turbulent flow simulation of a single-blade Magnus rotor
}

\author{
X. Bai ${ }^{1,2}$, C. Ji $3^{3^{*}}$ D, P. Grant ${ }^{4}$, N. Phillips ${ }^{4}$, U. Oza ${ }^{4}$, E. J. Avital ${ }^{1}$ and J. J. R. Williams ${ }^{1}$
}

\footnotetext{
* Correspondence: cnji@tju.edu.cn ${ }^{3}$ State Key Laboratory of Hydraulic Engineering Simulation and Safety, Tianjin University, Tianjin 300072, China

Full list of author information is available at the end of the article
}

\begin{abstract}
This paper presents numerical studies of the Magnus effect for a kinetic turbine on a horizontal axis. To focus on the Magnus blade, a single self-spinning cylindrical blade is assumed. An iterative direct-forcing immersed boundary method is employed within the Eulerian-Lagrangian framework due to its capability to treat complex and moving geometries. The Eulerian fluid domain is discretized using the finite volume method while the Magnus rotor is represented by a set of discrete points/markers. The aim of the numerical studies is to provide insights for the design process and predict aerodynamic performances under various operating conditions. Results for stationary and self-spinning cylinders in turbulent flows are found to be in good agreement with published data. By increasing the aspect ratio of the cylinder (simulated segment length over its diameter) from 3 to 10, a 30\% drop in lift coefficient and a $22 \%$ increase in drag coefficient were observed, which is believed to be attributed to an enhancement of the three-dimensionality of the near-wake. For the Magnus rotor, key parameters such as dynamic forcing and frequency, distribution of pressure coefficient and torque have been produced for two cases with different structural designs and working conditions. With increase of the aspect ratio from 3 to 10, stable forces are observed from the root side of the blade and the torque coefficient increases from 0.68 to 2.1 , which indicates a superior performance in terms of power extraction.
\end{abstract}

Keywords: Immersed boundary method, Large eddy simulation, Magnus effect, Spinning cylinder

\section{Introduction}

The Magnus effect is a method of producing lift forces around a rotating body when placed in the cross-flow of a fluid, and can be easily observed in a wide range of disciplines and applications, such as the curving motion of a spinning ball in various field sports. Compared with other lift generation devices, such as aerofoils, it has been claimed by many researchers that the Magnus effect force can reach much higher magnitudes given the same projection area and inflow velocity, and has no risk of stall [1, 2]. The general accepted view of Prandtl's limit on lift coefficient $\left(C_{\operatorname{Lmax}}=4 \pi\right)$ was exceeded from the experiment of a rotating cylinder by Tokumaru \& Dimotakis [3]. The reason for the violation is that Prandtl's limit is derived for two-dimensional flow

(c) The Author(s). 2021 Open Access This article is licensed under a Creative Commons Attribution 4.0 International License, which permits use, sharing, adaptation, distribution and reproduction in any medium or format, as long as you give appropriate credit to the original author(s) and the source, provide a link to the Creative Commons licence, and indicate if changes were made. The images or other third party material in this article are included in the article's Creative Commons licence, unless indicated otherwise in a credit line to the material. If material is not included in the article's Creative Commons licence and your intended use is not permitted by statutory regulation or exceeds the permitted use, you will need to obtain permission directly from the copyright holder. To view a copy of this licence, visit http://creativecommons.org/licenses/by/4.0/. 
but in reality, the three dimensional and end-effect of the rotating cylinder can result in an increase in generated lift [4].

Current research on the Magnus effect can be divided into several categories and the most extensively studied case is for a single cylinder rotating in the cross-flow. Mittal \& Kumar [4] reported the effect of velocity ratio on the lift coefficient and vortex generation in a laminar flow of $R e=200$. They also suggested that an increase of the aspect ratio of the cylinder (spanwise length/diameter) plays an important role in the increase of lift and the decrease of drag. Another proposition to improve the lift coefficient on a rotating cylinder was made by Thom [5], who suggested adding endplates to the cylinder which was later proved to be effective by the research of Badalamenti \& Prince [6].

Another area of interest that has been investigated for many years is the development of power generation devices based on the Magnus effect. The first engineering application of the Magnus rotor is the 'Flettner rotor' which was successfully trialled on a ship. Later, the 'Savonius rotor', which consisted of two semi-circular plates, was implemented in the design of wind turbines [7-9]. Akira et al. [10] produced a study on the classical horizontal-axiswind-turbine (HAWT) concept by replacing the aerofoil blades with Savonius rotors. The power coefficient reported from their experimental test in a wind tunnel is 0.075 for a Magnus rotor with five-cylinder blades. Although the power conversion efficiency is relatively small as compared with the traditional HAWTs, several advantages have been identified, such as less noise impact, lower rotational speed and longer durability, which have provided an open space for future development. The need for further research on Magnus kinetic turbine was also emphasized by Sedaghat [11] who developed a blade-element-momentum (BEM) approach that relies on pre-existing knowledge of the Magnus blade aerodynamic efficiency. A similar approach was also developed by Richmond-Navarro et al. [12]. Recently, Kazemi et al. [13] presented a novel design of a Magnus wind turbine. Instead of using a circular-section blade, they introduced a new blade section similar to NACA0021 airfoil with a rounded head and tail. Magnus effect was produced by circulating the blade's surface, and the maximum lift to drag ratio of 130 was achieved, proving the high efficiency and feasibility of the novel Magnus wind turbine design.

This paper aims to provide an insight of the performance of a Magnus blade of a horizontal axis kinetic turbine under different working conditions using a turbulent flow simulation investigation. Focus is given on the performance of a single blade and its key parameters, whereas, the interaction between the blades is left for a following study. Such an approach is also inherent in the widely-used BEM theory that corrects for the blade interaction only at a second stage [14]. The organization of the paper is as follows. First, the presented methodology is validated respectively for a stationary cylinder and a spinning cylinder in turbulent flows. The performance of a spinning cylinder with different aspect ratios is then investigated for two operating conditions with $A R=3$ and $10, R e=1.0 \times 10^{5}$, and $\alpha=2$. Further, a Magnus rotor rotating around a fixed axis while self-spinning is tested and key parameters, such as lift, drag and torque, are presented for discussion.

\section{Methodology and simulation setup}

\subsection{Governing equations}

The numerical simulations are carried out using CgLES-IBM $[15,16]$, which is an inhouse Computational Fluid Dynamic (CFD) C code designed for fluid-structure 
interaction problems. This code has been used for many years by several researchers on high-end computing facilities and is highly parallelized and efficient. Recent applications include sediment entrainment in turbulent channel [17, 18], red blood cell aggregation $[19,20]$, marine current turbine [21], and vortex-induced vibration of cylinders [22-27].

The code solves the incompressible viscous single phase Navier-Stokes equations:

$$
\partial_{t} \mathbf{u}+(\mathbf{u} \cdot \nabla) \mathbf{u}+\nabla p=v \nabla^{2} \mathbf{u}+\mathbf{f},
$$

and the continuity equation,

$$
\nabla \cdot \mathbf{u}=0
$$

where $\mathbf{u}$ and $p$ are the fluid velocity and pressure, $v$ is the kinematic viscosity and $\mathbf{f}$ is a volume force term (gravitational force and feedback force). The Eulerian fluid domain is discretized with a second order finite volume method with a staggered Cartesian grid. To decouple the velocity and pressure field, the two-step 'predictor-corrector' projection method is employed. The third-order Runge-Kutta scheme is used for time advancement.

An iterative direct-forcing immersed boundary method [15] has been adopted to represent the solid body by a set of discrete points, which are allowed to move either at a given speed (as in this study) or by surrounding dynamic forces. In contrast with the traditional approach that uses body-conformal meshes, the current scheme requires little effort in mesh generation, and can be applied to a wide range of problems, especially those with complex flows and geometries. The communication of velocity and forces between the surrounding fluid and solid bodies are achieved by setting a boundary condition on a set of Lagrangian points/markers. For a viscous fluid, this can be expressed as

$$
\mathbf{U}=\mathbf{V}
$$

where $\mathbf{U}=I(\mathbf{u})$ is the interpolated velocity from the surrounding flow field using a delta function $I$ and $\mathbf{V}$ is the fixed velocity given as boundary condition. Through the interpolation, the physical field from the Eulerian domain is projected to each individual point/marker and the feedback force can be computed as the difference between the desired velocity $(\mathbf{V})$ and interpolated velocity $(\mathbf{U})$. Then, the calculated feedback force is mapped back to the Cartesian grids as a body force via the same delta function. Overall, the present scheme fits in the framework of Eulerian (fluid)-Lagrangian (solid) system. Another advantage of the proposed scheme is the ability to employ fast Poisson solver with the structured arrangement of Cartesian grids. In the present study, the BiCGSTAB solver together with a geometric multi-grid pre-conditioner was used to solve the pressure Poisson equation, which was successfully applied to the studies of a horizontal axis marine turbine $[21,28]$.

To simulate turbulent flow, large eddy simulation (LES) is carried out using a MixedTime-Scale (MTS) sub-grid model [29]. The Sub-Grid Scale (SGS) model uses fixed model-parameters and is constructed within the concept of a mixed time-scale, where the eddy viscosity $\left(v_{t}\right)$ is expressed by (velocity scale $)^{2} \times($ time scale). And the velocity scale can be obtained from the turbulent kinetic energy $k_{e s}$, 


$$
k_{e s}=(\overline{\mathbf{u}}-\widehat{\overline{\mathbf{u}}})^{2},
$$

where $\widehat{\overline{\mathbf{u}}}$ is the filtered velocity by the Simpson rule. The time scale can be calculated using the harmonic average of $\Delta / \sqrt{k_{e s}}$ and $1 /|\bar{S}|$ :

$$
T_{S}^{-1}=\left(\Delta / \sqrt{k_{e s}}\right)^{-1}+\left(C_{T} /|\bar{S}|\right)^{-1},
$$

where $\Delta$ is the averaged grid spacing and $\bar{S}$ is the strain-rate tensor computed from the filtered velocity field. $C_{T}=10$ is a parameter based on the a-priori tests by Inagaki et al. [29]. The turbulent viscosity can be calculated as:

$$
v_{t}=C_{M T S} k_{e s} T_{S}^{-1},
$$

where $C_{M T S}$ is a parameter set to 0.05 , as in Inagaki et al. [29]. In the near wall region, the velocity scale is close to zero and the time scale is averaged to a shorter scale, so no wall-damping function is needed.

\subsection{Simulation setup for the Magnus rotor}

In Fig. 1, the rotor is shown rotating counter-clockwise in the $X-Y$ plane while the cylinder itself is spinning counter-clockwise in the $y$ axis. The spinning of the cylinder is expected to generate positive force in the direction of the rotor rotation. With the rotation of the Magnus rotor, the additional lift force on the blade is used to provide torque, and hence can be used as a power extraction device, like a horizontal axis kinetic turbine. Two sets of coordinate systems are employed: a global coordinate system to keep track of the location of the rotor and a local coordinate system fixed on the cylinder to take account of the self-spinning. At a given time $T$, the angle rotated by the rotor from its initial location can be determined by $\phi=\Omega T$, and the global coordinates of the immersed boundary points $\left(X_{c}, Y_{c}, Z_{c}\right)$ on the rotor can be updated accordingly.

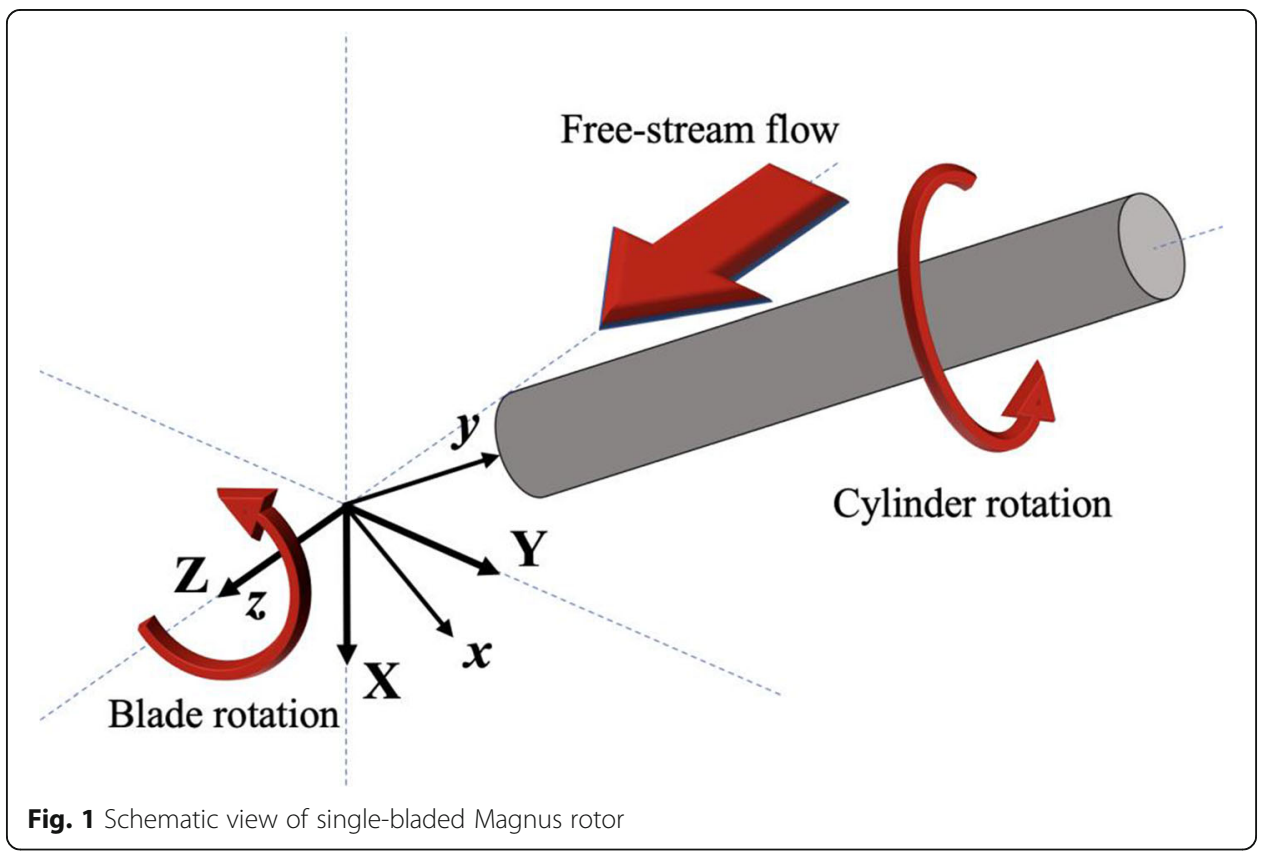


The local coordinate system is initially set to be the same as the global system and rotates against the $Z$-axis, in which the location of the Lagrangian markers $\left(x_{c}, y_{c}, z_{c}\right)$ remains unchanged at all times. The final desired velocity can be obtained by considering both the rotor rotation and self-spinning of the cylinder, which is used to obtain the dynamic forcing and to correct the surrounding flow field. We write $\mathbf{u}_{r}$ for the velocity vector of rotor rotation and $\mathbf{u}_{c}$ for the velocity vector of self-spinning, and the velocity boundary condition on each of the Lagrangian markers can be obtained by

$$
\mathbf{V}=\left(\begin{array}{cc}
u_{r}+u_{c} & -Y_{c} \Omega+z_{c} \omega \cos (\phi) \\
v_{r}+v_{c}
\end{array}\right)=\left(\begin{array}{cc}
\left.X_{c} \Omega+z_{c} \omega \sin (\phi)\right) \\
w_{r}+w_{c} & -x_{c} \omega
\end{array}\right.
$$

\subsection{Calculation of dynamic loading}

The force vector $\mathbf{F}$ on the rotor blade is set as the sum of the forces on all the markers over the exposed faces and is calculated in three directions. Using the global coordinate system shown in Fig. 1, the lift force $\left(F_{L}\right)$ is in the $-X$ direction, drag force $\left(F_{D}\right)$ is in the $+Z$ direction, and $Y$ is a kind of 'axial' force $\left(F_{A}\right)$. To investigate the dynamic forcing on the spinning cylinder, the global drag, lift and axial forces were then transformed to the local coordinate system at each time instance. Using the notation specified above, the local forces can be calculated as:

$$
\begin{aligned}
& f_{l}=F_{L} \cos \phi+F_{A} \sin \phi, \\
& f_{d}=F_{D}, \\
& f_{a}=-F_{L} \sin \phi+F_{A} \cos \phi .
\end{aligned}
$$

The dimensionless force coefficients for the Magnus blade are evaluated by the commonly adopted definition for the case of flow over a cylinder. The coefficients for drag, lift and axial force are specified as below:

$$
\begin{aligned}
& C_{d}=\frac{f_{d}}{\frac{1}{2} \rho U_{\infty}^{2} D L}, \\
& C_{l}=\frac{f_{l}}{\frac{1}{2} \rho U_{\infty}^{2} D L}, \\
& C_{a}=\frac{f_{a}}{\frac{1}{2} \rho U_{\infty}^{2} \frac{\pi D^{2}}{4}} .
\end{aligned}
$$

The torque on the Magnus blade is computed using the local force and coordinates. Summing over all Lagrangian points, the torque and its dimensionless coefficient can be evaluated by,

$$
t_{s}=\sum_{I B P s}\left(f_{l} \cdot y_{c}-f_{a} \cdot x_{c}\right),
$$




$$
C_{t_{s}}=\frac{t_{s}}{\frac{1}{2} \rho U_{\infty}^{2} D L^{2}} .
$$

For an operating Magnus rotor, the power coefficient can then be computed using the rotor angular speed, rotor diameter and free stream velocity,

$$
C_{\text {power }}=\frac{t_{s} \Omega}{\frac{1}{2} \rho U_{\infty}^{3} \frac{\pi D_{c}^{2}}{4}} .
$$

\section{Results for a single spinning cylinder}

To show the accuracy of the adopted numerical methodology, two validation cases were selected for an infinite length cylinder in turbulent flow with a Reynolds number $R e=1.4 \times 10^{5}$ and velocity ratios $\alpha=0.0$ and 2.0. Moreover, a mesh sensitivity study was carried out to investigate the impact of the grid resolution around the cylinder for $\alpha=2.0$. Current results showed broadly satisfactory agreement for all the validation cases as compared with available experimental measurements and numerical data. Details of the validation cases are in Appendix.

In this section, the effect of the aspect ratio $(A R=L / D)$ on the key performance characteristics is numerically investigated for a spinning circular cylinder with finite length in turbulent flow at $R e=1.0 \times 10^{5}$ and $\alpha=2.0$. From the study of Breuer [30] on turbulent flow past a stationary cylinder, the aspect ratio is considered as one of the most influencing parameters that cause the differences between experimental measurements and computed results. However, to use Magnus effect for power generation purpose, the effect of the aspect ratio is yet to be studied.

Two designs of cylinder were selected for the numerical studies with different aspect ratios and spin speed, as specified in Table 1. The parameters are given in engineering units and then normalized by the cylinder's diameter and free-stream velocity. The dimensionless Reynolds number and velocity ratio for both cases are the same at $R e=$ $1.0 \times 10^{5}$ and $\alpha=2.0$. The cylinder has a circular section in the $X-Y$ plane, with an axis aligned with the $Z$ direction. In the $X-Y$ plane, the computational box has the dimensions of $[-7.8 D, 17.8 D] \times[-12.8 D, 12.8 D]$, while in the $Z$ direction the span-wise dimension of the computational domain equals to the cylinder's length. The Dirichlet boundary condition is used at the inflow $(-X)$, together with free-slip top $(+Y)$ and bottom $(-Y)$ boundary conditions. A Neumann-type boundary condition is adopted at the outflow $(+X)$, and periodicity is imposed in the span-wise direction. The computational domain is discretized on a stretched Cartesian mesh with a grid resolution of $256 \times$ 256 in the $X-Y$ plane. An embedded uniform sub-mesh with spacing of $D / 64$ around the cylinder is employed. Uniform meshes are adopted in the $Z$ direction with the number of span-wise grids being 192 and 96 for Cases 1 and 2, respectively. The time

Table 1 Computational parameters of the single spinning cylinder

\begin{tabular}{llllllll}
\hline Cases & $\boldsymbol{A} \boldsymbol{R}$ & $\mathbf{R e}$ & $\boldsymbol{D}(\mathbf{m})$ & $\boldsymbol{L}(\mathbf{m})$ & $\boldsymbol{\omega}(\mathbf{r a d} / \mathbf{s})$ & $\boldsymbol{a}$ & $\boldsymbol{U}_{\infty}(\mathbf{m} / \mathbf{s})$ \\
\hline 1 & 10 & $10^{5}$ & 0.3 & 3.0 & 66.67 & 2.0 & 5 \\
2 & 3 & $10^{5}$ & 1.0 & 3.0 & 6.00 & 2.0 & 1.5 \\
\hline
\end{tabular}


step size was chosen to make the maximum Courant number approximately equal to 0.35 for both cases.

Table 2 shows the comparison of drag and lift coefficients for the two cases. Although the Reynolds number and velocity ratio were the same, significant differences are present in the drag and lift forces and their r.m.s. values, indicating an aspect ratio dependency. It is noted that with the increase of the aspect ratio the Magnus effects become weaker, i.e., higher drag and lower lift forces. With an increase in $A R$ from 3.0 to 10.0, the mean drag coefficient $\bar{C}_{d}$ grows from 0.49 to 0.60 , showing an increase of $22 \%$, approximately. While the mean lift coefficient $\bar{C}_{l}$ decreases from 4.17 to 2.90 , showing a drop of $30 \%$, approximately. The same behaviour is also observed in Breuer's [30] study on a stationary cylinder, where results for $A R=2.0$ showed about $20 \%$ increase in drag coefficient as compared to $A R=1.0$. For spinning cylinder, the $3 \mathrm{D}$ URANS study by Craft et al. [31] for the same Reynolds number and velocity ratio as the present study showed no aspect ratio dependency for $A R=1.0$ and 3.0. However, a more recent LES study by Rolfo \& Revell [32] reported both the absolute lift and drag coefficients drop by nearly $10 \%$ when the aspect ratio increases from $A R=1.0$ to $A R=$ 2.0 at $R e=5.0 \times 10^{5}$ and $\alpha=5.0$. For results with $A R=0.5$ and $A R=1.0$, with the expansion in the spanwise dimension, the drag coefficient increased mildly by $3 \%$ and the lift coefficient decreased by about $10 \%$.

One possible reason of the observed aspect ratio dependency can be attributed to the three-dimensionality of the near-wake behind the spinning cylinder. This argument is further confirmed by the contours of the instantaneous stream-wise velocity in the near-wake, see Fig. 2, in which a clear alternating pattern is observed for the case of $A R=10$. The three-dimensional instabilities with increased aspect ratio contribute to the increase of drag and loss in lift, which is difficult to capture in the previously mentioned URANS simulations. The mean pressure contours and the streamlines computed using the averaged velocity in Fig. 3 clearly show the differences between the two cases. For the case of $A R=10$, the upper recirculation bubble disappears and the lower one is compressed due to the averaging along the span-wise direction, which increases the smoothness of the vorticity strength in the three-dimensional near-wake. For the case of $A R=3$, the low-pressure region near the top of the cylinder is significantly larger than that with $A R=10$, which explains the larger mean lift for the case of $A R=3$. Moreover, two recirculation bubbles are observed close to the cylinder, indicating that the wake is still strongly affected by the alternate vortex shedding. This could be used to explain the stronger force fluctuations (r.m.s values) observed for the case of $A R=3$. Nevertheless, the aerodynamic forces acting on the cylinder will still be higher with extended cylinder length although the benefit could be offset by the additional power required to maintain the spinning speed of the cylinder. Further investigation is still

Table 2 Comparison of drag and lift coefficients of the single spinning cylinder with different aspect ratios

\begin{tabular}{llllll}
\hline Cases & $\boldsymbol{A} \boldsymbol{R}$ & $\overline{\boldsymbol{C}}_{\boldsymbol{d}}$ & $\boldsymbol{C}_{\boldsymbol{d}, \boldsymbol{r m s}}$ & $\overline{\boldsymbol{C}}_{\boldsymbol{l}}$ & $\boldsymbol{C}_{\boldsymbol{l}, \boldsymbol{r m s}}$ \\
\hline 1 & 10 & 0.60 & 0.035 & 2.90 & 0.10 \\
2 & 3 & 0.49 & 0.084 & 4.17 & 0.155 \\
\hline
\end{tabular}




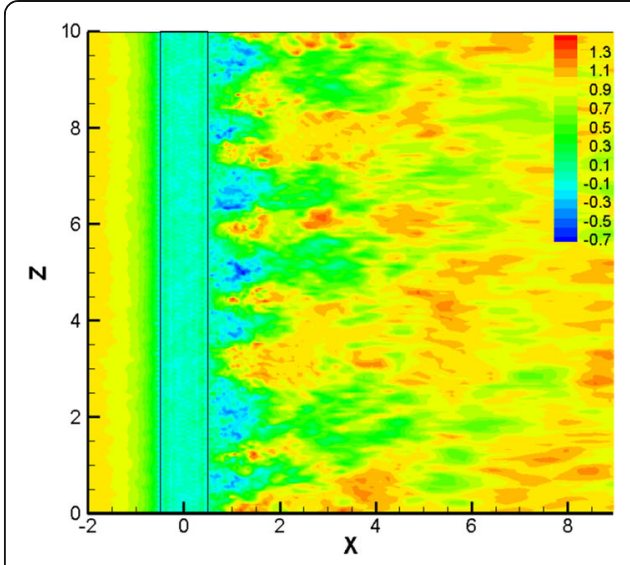

(a)

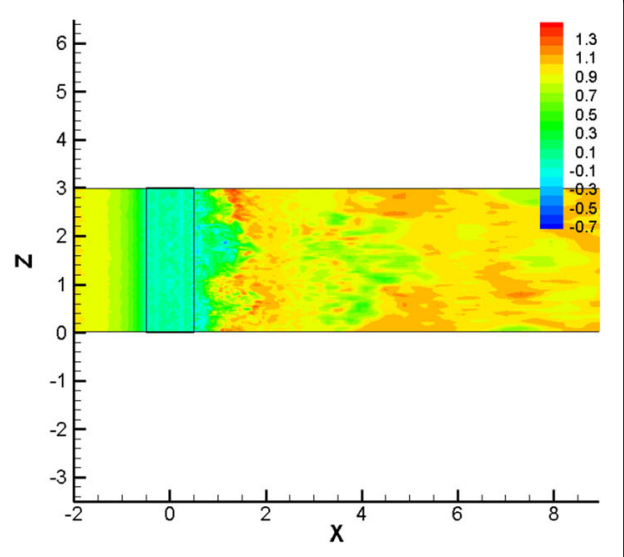

(b)

Fig. 2 Contours of dimensionless instantaneous stream-wise velocity of (a) $A R=10$ and (b) $A R=3$

required to fully understand the implications of these findings and propose an optimal design.

\section{Results for a single-blade Magnus rotor}

To explore the possibility of using the Magnus effect for power generation, the concept of a horizontal axis kinetic turbine is adopted. To focus on the Magnus blade, a single self-spinning cylindrical blade is assumed. Two designs of the Magnus blade are presented in Table 3, with the key parameters defined in engineering units. The Reynolds number based on the free stream velocity and the diameter of the cylindrical blade is $1.0 \times 10^{5}$ and a constant velocity ratio of 2.0 is set for both cases to match the previous study of flow past a spinning cylinder. The rotor's diameter $D_{c}$ is $7.0 \mathrm{~m}$ and the rotational centre is located at $(0,0,0) \mathrm{m}$. The size of the computation domain is set as [$22.925,22.925] \mathrm{m},[-22.925,22.925] \mathrm{m}$ and $[-9.075,33.050] \mathrm{m}$ in the $X, Y, Z$ directions respectively. Normalized by the rotor diameter, the computation domain equals to $6.55 D_{c} \times 6.55 D_{c} \times 6 D_{c}$ in three directions. The computational domain is discretized on a stretched Cartesian mesh with special attention given to the uniform sub-mesh

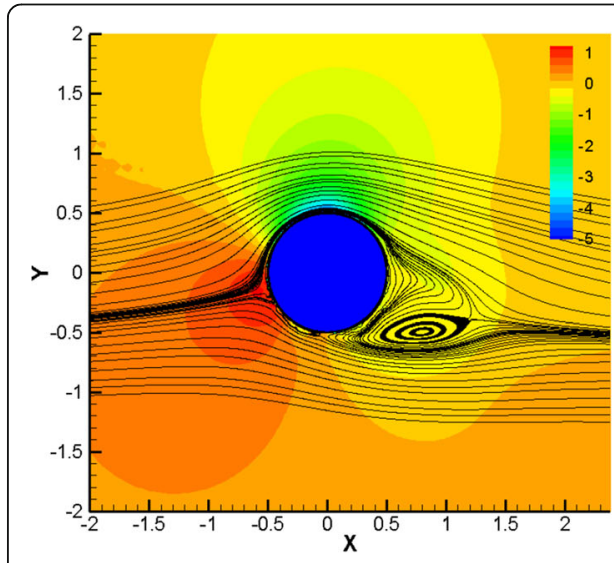

(a)

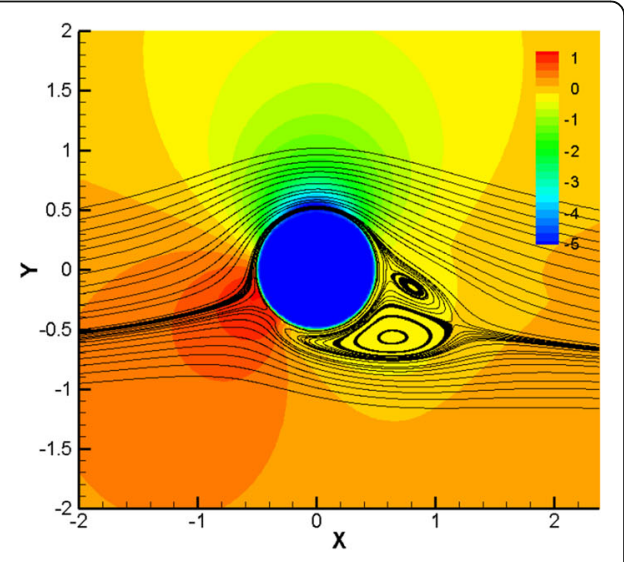

(b)

Fig. 3 Contours of the dimensionless pressure coefficient and streamlines for (a) $A R=10$ and (b) $A R=3$ 
Table 3 Simulation parameters for the single-blade Magnus rotor

\begin{tabular}{llllllll}
\hline Cases & $\boldsymbol{A R}$ & $\boldsymbol{U}_{\boldsymbol{\infty}}(\mathbf{m} / \mathbf{s})$ & $\boldsymbol{D}(\mathbf{m})$ & $\boldsymbol{L}(\mathbf{m})$ & $\boldsymbol{L}_{\boldsymbol{r}}(\mathrm{m})$ & $\boldsymbol{\Omega}(\mathrm{rad} / \mathbf{s})$ & $\boldsymbol{\omega}(\mathbf{r a d} / \mathbf{s})$ \\
\hline 3 & 10 & 5.0 & 0.3 & 3.0 & 0.5 & 1.40 & 66.67 \\
4 & 3 & 1.5 & 1.0 & 3.0 & 0.5 & 0.32 & 6.00 \\
\hline
\end{tabular}

region covered by the rotation of the blade. For Case 3, the total mesh number is 1024 $\times 1024 \times 256$ points with the finest spacing of $D / 40$. For Case 4 , the total mesh number is $512 \times 512 \times 256$ with the finest spacing of $D / 80$.

\subsection{Magnus rotor simulation with $A R=10$}

The Magnus rotor with the aspect ratio of 10, self-spinning angular speed of $66.67 \mathrm{rad} /$ $\mathrm{s}$ and rotor rotation speed of $1.40 \mathrm{rad} / \mathrm{s}$ (Case 3) was run in the computation domain specified above until the simulation reached a statistically steady state. The time step size was chosen to make the maximum Courant number approximately 0.7. The dynamic forces acting on the blade were monitored throughout the simulation and then transformed to the local coordinates to compute the dimensionless force coefficient. The spectra analysis of the recorded data, see Fig. 4, indicates the presence of a dominating frequency for both the lift and drag coefficients. Interestingly, the observed frequency is equivalent to each other which is not observed for flow over a solely spinning cylinder. The imposed motion of rotation of the Magnus rotor is believed to contribute to the change of the flow field and hence the dynamic loading on the blade, introducing more low-frequency oscillations. The simulation was restarted for another 5 rotor rotation cycles while the time averaged statistics were gathered.

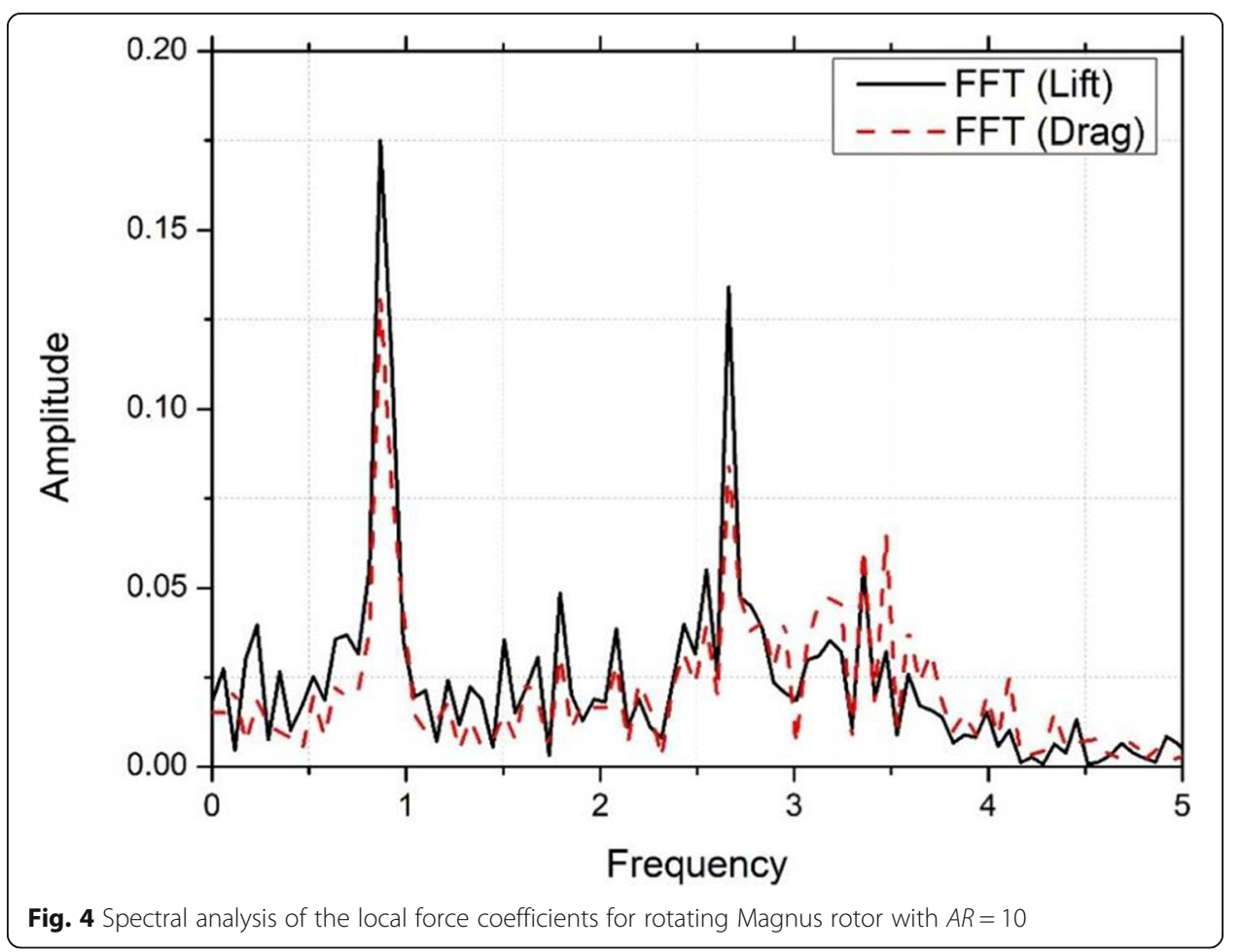


Table 4 lists the computed force coefficients and their variance. Referring to the solely spinning case in Section 3, the r.m.s values of the coefficients in the present simulation increase dramatically, i.e., two times for the lift coefficient and more than five times for the drag coefficient. With the rotation of the rotor, the mean value of the lift coefficient on the cylinder blade is 3.18 , slightly larger than the value of 2.90 for a single spinning cylinder. The drag coefficient experiences a more noticeable jump, indicating stronger resistance to the incoming flow due to the motion of rotation. One possible explanation can be derived by looking at the incidence angle between the inflow and the surface of the blade. Considering the free stream inflow and the movement of the blade, the combined inflow will no longer be normal to the front surface of the cylinder but is diverted towards the lower surface (assume the cylinder is spinning anticlockwise), hence the lift force will be shifted anti-clockwise by the same angle, resulting in higher drag and smaller lift. Additionally, the motion of rotation will increase the magnitude of the relative inflow, leading to higher absolute forces. For the current case, the tip speed ratio $\left(T S R=\frac{D_{c} \Omega}{2 U_{\infty}}\right)$ is about 1 , which could offset the incidence angle by $45^{\circ}$. This argument is further confirmed by the distribution of pressure coefficient along the spanwise of the blade.

Figure 5 shows the contours of dimensionless pressure coefficient on the Magnus blade. The top plot corresponds to the surface of the cylinder facing the inflow, where a high-pressure region has formed as the oncoming flow hits the rotating blade. A closer examination shows the distribution of high-pressure regions is not uniform or straight along the spanwise direction. This is clearly due to the global motion of rotation. The surface closer to the tip has the largest $C_{p}$ value while from root to the midspan, the peak value of $C_{p}$ shows very small variations. On the back side of the cylinder, a region of low-pressure concentration is observed at the mid-span and extends towards the tip of the blade. The largest absolute value of the negative $C_{p}$ is located slightly downstream of the middle of the blade, unlike the front surface. Further away from the bottom face of the cylinder $\left(\theta=90^{\circ}\right)$, a scattered distribution of high and low is observed, indicating oscillations induced by flow turbulence.

The pressure coefficient at different spanwise locations on the Magnus blade is shown in Fig. 6 for $y=0.1 L, y=0.5 L$ and $y=0.9 L$ respectively. As expected, the base point of the pressure curve is shifted anticlockwise to a larger azimuthal angle at all three locations. The largest angle is observed at about $\theta=140^{\circ}$ for $y=0.9 \mathrm{~L}$ as compared to $90^{\circ}$ for flow past a single spinning cylinder. Among the three curves, the largest absolute value of negative $C_{p}$ is found at $y=0.5 \mathrm{~L}$, while the lowest is at $y=0.1 \mathrm{~L}$, which is closer to the root of the blade. All the findings indicate the global motion of rotation has significantly altered the flow field hence the pressure distribution on the Magnus blade. As the pressure is expected to account for most of the force generated, the current study indicates the main contribution for the lift force is around the middle of the blade while the drag force is more dominant near the tip.

Table 4 Comparison of local drag and lift coefficients of the rotating Magnus rotor with different aspect ratios

\begin{tabular}{lllllll}
\hline Cases & $\bar{C}_{d}$ & $\boldsymbol{C}_{\boldsymbol{d}, \text { rms }}$ & $\bar{C}_{\boldsymbol{l}}$ & $\boldsymbol{C}_{\boldsymbol{l} \text {, rms }}$ & $\overline{\boldsymbol{C}}_{a}$ & $\boldsymbol{C}_{\boldsymbol{a}, \boldsymbol{r m s}}$ \\
\hline 3 & 3.74 & 0.194 & 3.18 & 0.235 & 2.23 & 0.27 \\
4 & 3.48 & 0.13 & 1.33 & 0.086 & 1.413 & 0.094 \\
\hline
\end{tabular}




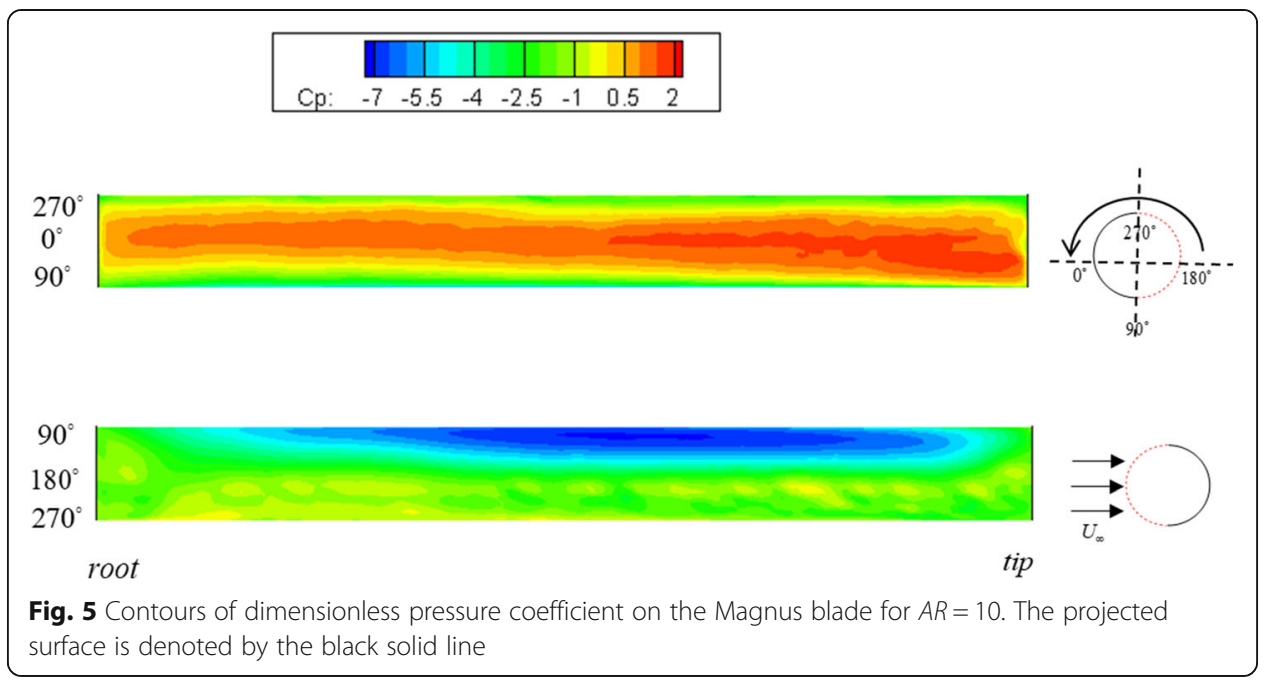

Having obtained the dynamic forces, the torque on the Magnus rotor was calculated using Eq. 14 and then projected to the surfaces of the blade, see Fig. 7. Note the negative value of torque is in the same direction of the global rotation. The top plot corresponds to the lower face of the cylinder, where the torque is uniformly distributed in the first half of the blade (root side) and an alternating pattern is observed on the other half (tip side). Looking at the upper face of the cylinder, the weaker but clearly visible alternating pattern is also observed along the spanwise direction. In terms of the magnitude, the largest values of both negative and positive torque are found near the tip of the blade, indicating stronger instability. This behaviour can also be explained by the increased inflow velocity and incidence angle induced by the global motion of rotation. Integrating along the span of the Magnus blade, the major contribution of the total torque still comes from the side, where stable forces are present.

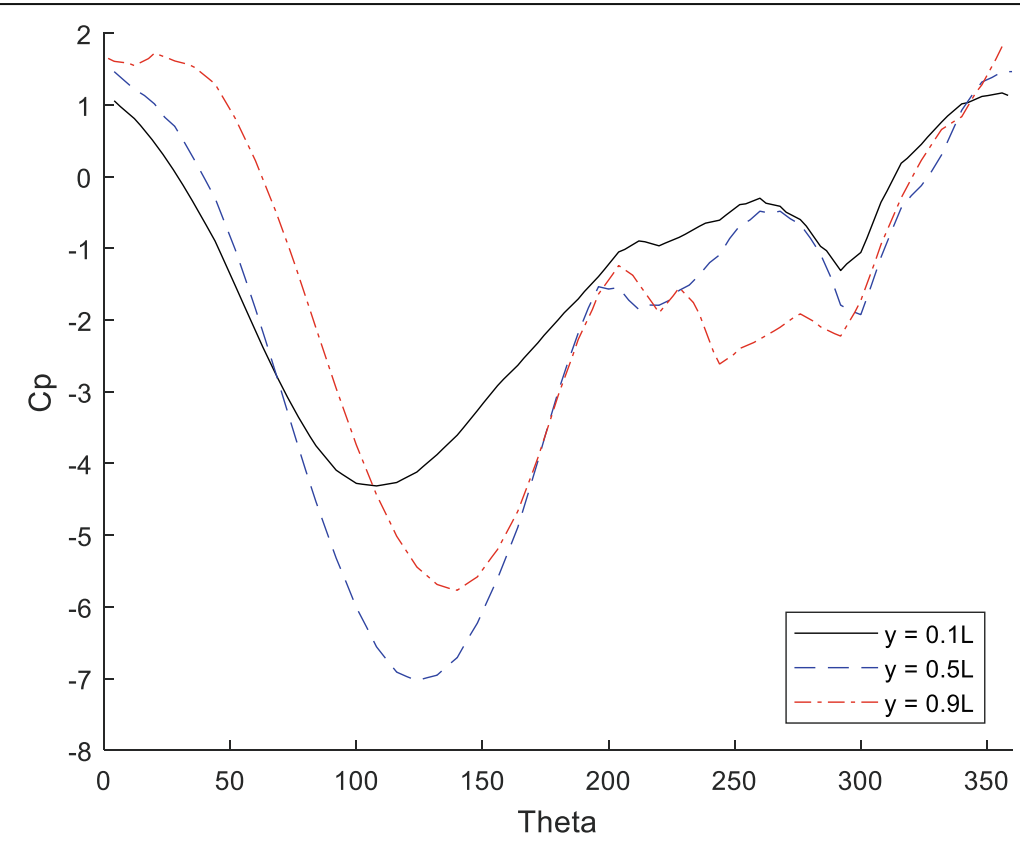

Fig. 6 Distribution of pressure coefficient on the Magnus blade at different spanwise locations for $A R=10$ 


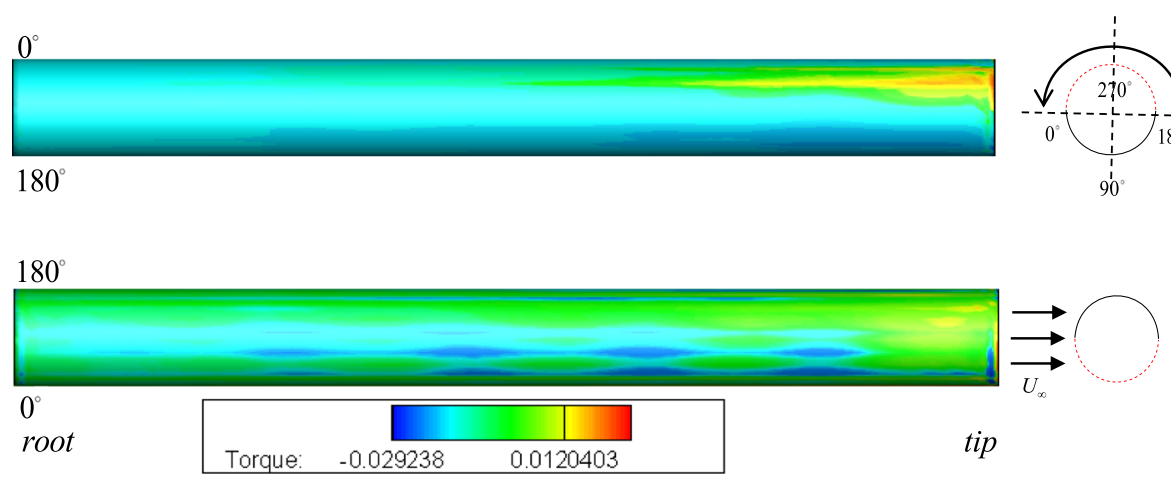

Fig. 7 Distribution of torque on the Magnus blade with $A R=10$. The projected surface is denoted by the black solid line

Large scale vortical turbulent structures, shed by the flow over a cylinder can be effectively identified using the $\mathrm{Q}$ values, e.g. Yu et al. [33]. Figure 8 shows the instantaneous $\mathrm{Q}$ criterion in the vicinity of the blade. A clear track of the vortices can be seen from the path of the rotation. Notably, the formed vortices tend to get detached from only one side of the cylinder and shed downstream before quickly breaking down into smaller structures. On the other side of the cylinder, a large structure remains attached to the blade surface, where the surrounding flow has the highest streamwise velocity. This large structure can be directly related to the low-pressure concentration as shown earlier in Fig. 5 and responsible for the generation of Magnus lift. At the tip of the Magnus rotor, the formation of the tip vortices is clearly visible and interferes with the vortices formed on the cylinder's body before shedding downstream in a spiral manner. The unsteady behaviour strengthens the oscillations of the forcing closer to the tip and hence leads to the alternating pattern in the torque shown in Fig. 7.

\subsection{Magnus rotor with $\mathrm{AR}=3$}

Numerical results for the Magnus rotor with a second blade design are presented as below. The blade with $A R=3$ is rotated at a fixed angular speed of $0.32 \mathrm{rad} / \mathrm{s}$ and the velocity ratio is kept as 2.0 to match the previous study. Figure 9 shows the spectral analysis of the fluctuations of the local lift and drag coefficients. A matching dominating frequency is observed for the two force coefficients, leading to the same behaviour as the case with $A R=10$. Table 5 presents the observed frequency for both cases. With $A R=3$, the dominating frequency is of $0.2 \mathrm{~Hz}$, while with $A R=10$ and angular speed of $1.40 \mathrm{rad} / \mathrm{s}$, a frequency of $0.868 \mathrm{~Hz}$ is obtained. If we define a new Strouhal number using the frequency of dynamic loadings $(f)$, rotor diameter $\left(D_{c}\right)$ and blade rotation speed $(\Omega)$,

$$
S t_{D_{c}}=\frac{f_{s} D_{c}}{\frac{1}{2} \Omega D_{c}}=\frac{2 f_{s}}{\Omega} .
$$

The value for Case 3 is 1.24 and for Case 4 is 1.25 , which indicates that the rotation of the Magnus rotor is the driving parameter for the change of the dominant frequency of the lift and torque variation. From the present findings, the dominating frequency 


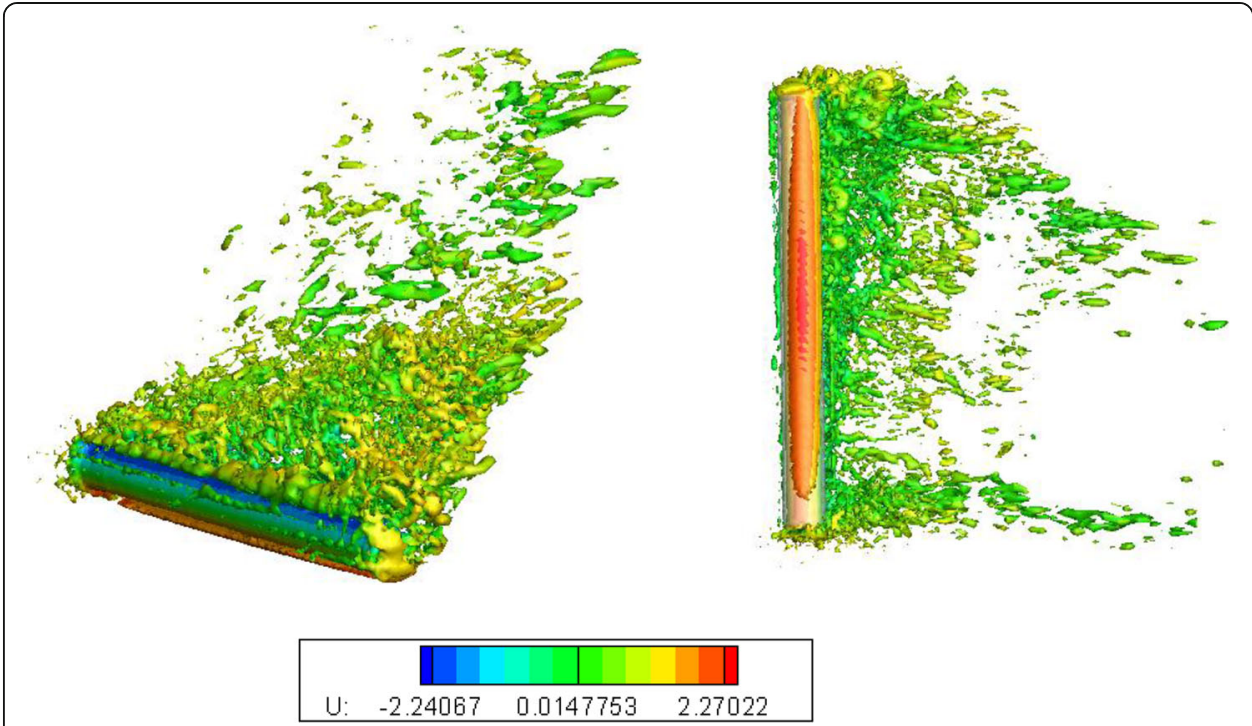

Fig. 8 Instantaneous $Q$ criterion $(Q=1000)$ for Magnus rotor with $A R=10$. Isosurface is colored by the normalized streamwise velocity $\left(U / U_{\infty}\right)$

for the dynamic forces depends only on the angular speed of global rotation, irrespective of the design of the blade. This finding can serve as an important point in the structural design of Magnus turbines. For the mean coefficients' value, see Table 4, the current case shows an increase of the drag coefficient as compared to the solely spinning cylinder, as the previous case with $A R=10$. The lift coefficient reported in the current case drops to 1.33 , indicating an inferior performance for the lift-to-drag ratio. This behaviour is explored in more detail with the distribution of the pressure coefficient.

Figure 10 shows the contours of dimensionless pressure coefficient on the Magnus blade with $A R=3$. Generally speaking, the distribution of the high-pressure region and low-pressure region is very similar to the results presented for the previous case of $A R=10$. A closer examination on the negative pressure region shows its centre has shifted towards the tip of the blade and downwards towards a larger azimuthal angle. More importantly, the magnitudes of the negative pressure are smaller than the previous case, which could be the reason for the drop in lift coefficient. The pressure coefficient at $y=0.1 \mathrm{~L}, y=0.5 \mathrm{~L}$ and $y=0.9 \mathrm{~L}$ is shown in Fig. 11, where the absolute values of the base pressure coefficient at different locations are all reduced by a dimensionless amount of 0.5 to 1.0. The azimuthal angle of the base pressure at $y=0.5 \mathrm{~L}$ and $y=0.9 \mathrm{~L}$ coincides with each other, indicating a larger relative incidence angle at the middle of the blade. The weaker pressure difference and the shift of the base pressure will have combined influence on the reduction of lift forces. However, it is not yet clear which factor is more dominant.

Figure 12 shows the distribution of torque on the Magnus blade with $A R=3$. Along the extension of span, a clear alternating pattern is observed on the top surface as well as the bottom surface. As the torque is directly related to lift, it is expected that a stable force is no longer present at the root of the blade. The largest values of both negative and positive torque are still found to be near the tip, owing to the large incidence angle. Interestingly, oscillations of the torque are also found near the root of the blade, indicating the presence of the end effect. 


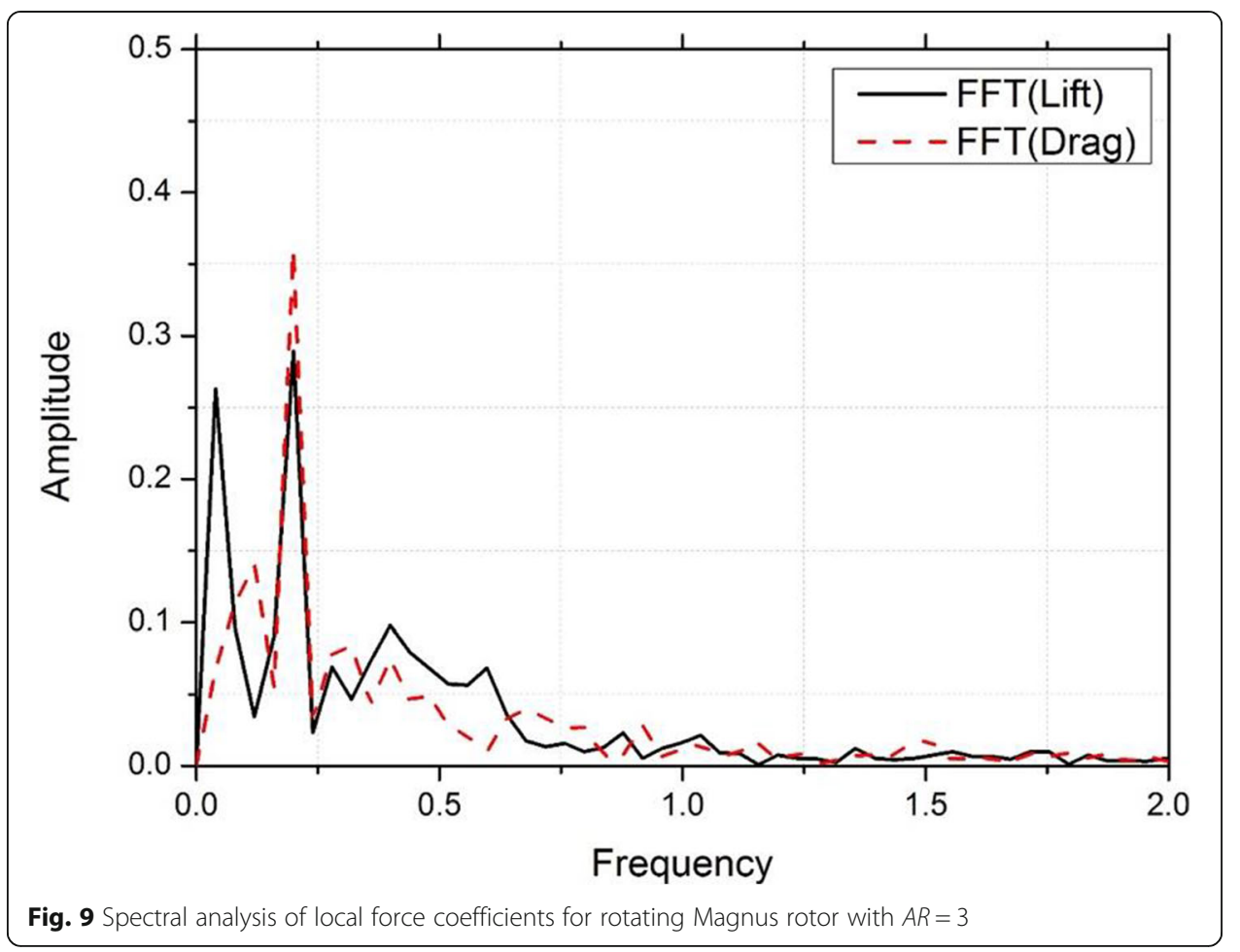

Figures 13 shows the instantaneous $\mathrm{Q}$ criterion near the blade with $A R=3$. It is noticed that the iso-surfaces of vortices have a twisted shape in the middle of the cylinder which is attributed to the self-spinning of the cylinder. At the upper half, where the rotor rotation is the dominant factor for vortex formation, the vortices are shed in a spiral manner following the track of rotation, while at the bottom half, where the selfspinning is more dominant, the vortices tend to follow the spinning direction. The previously observed large structure at the suction side is not captured in the present study, probably suppressed with the selection of the current aspect ratio.

The power coefficient for the investigated designs of Magnus rotor is shown in Table 5. Among the two cases, the case with $A R=3$ seems to show better performance with a power coefficient of $0.0509,23 \%$ higher than the case with $A R=10$. However, these values can be misleading to determine the optimal design of Magnus rotors, because the denominator in the classical calculation for the power coefficient, i.e., Eq. 16, considers the whole region swept by the rotation of the blades, including the area from the axis of rotation to the root of the cylinder. A more representative parameter is the dimensionless torque coefficient, the production of torque per unit length of the blade, i.e., Eq. 15. The computed torque coefficient for the case with $A R=10$ is 2.1 and the value for the case with $A R=3$ is 0.68 . Clearly, this indicator reflects the superior behaviour of Case 3 as we previously witnessed in the mean force coefficients.

Table 5 Comparison of dominant fluctuation frequency and dimensionless torque and power coefficient of the rotating Magnus rotor with different aspect ratios

\begin{tabular}{llll}
\hline Cases & $\boldsymbol{f}$ & $C_{t_{s}}$ & $\boldsymbol{C}_{\text {power }}$ \\
\hline 3 & 0.868 & 2.1 & 0.0413 \\
4 & 0.20 & 0.68 & 0.0509 \\
\hline
\end{tabular}




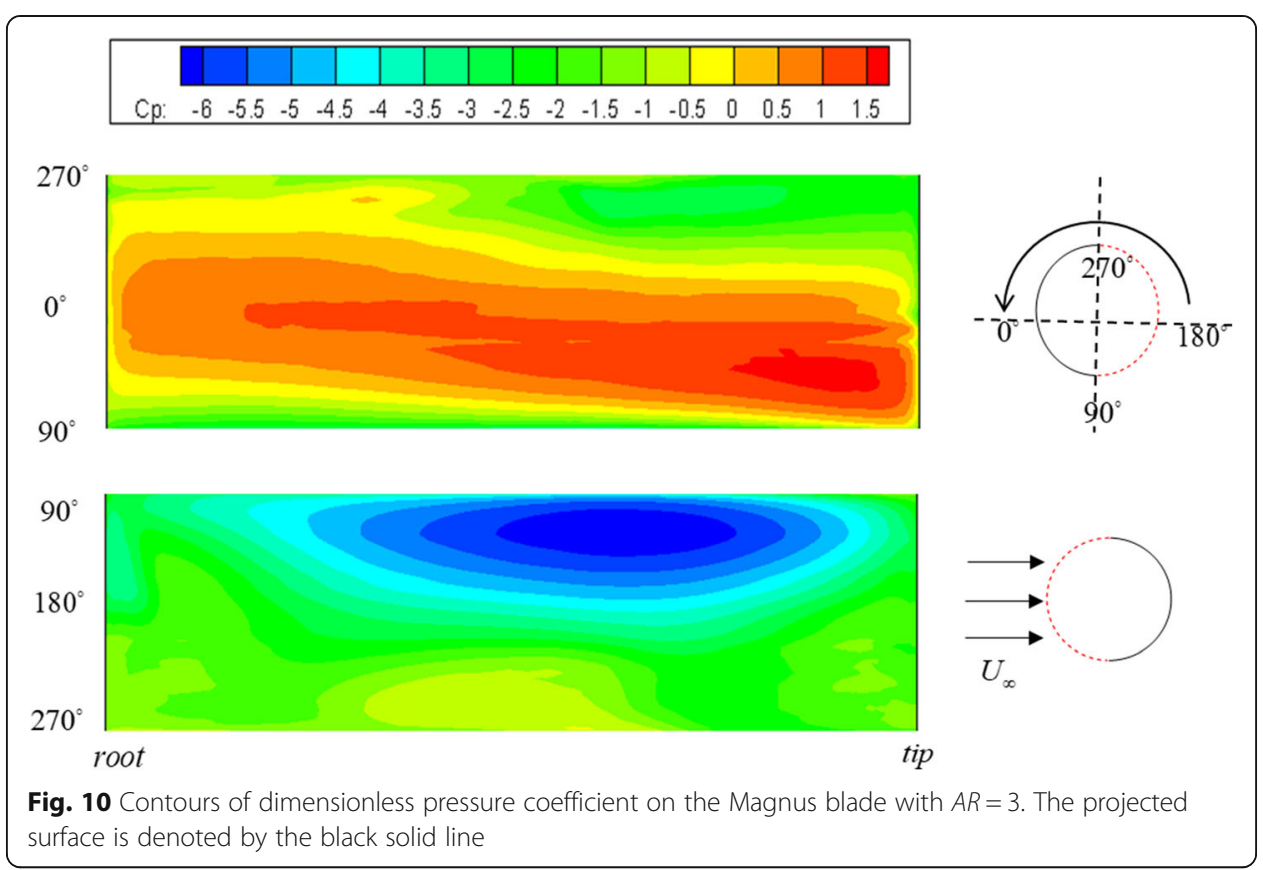

\section{Conclusions}

In this paper, the idea of using a Magnus rotor as a power extraction device was numerically investigated using the direct forcing Immersed Boundary Method and Large Eddy Simulation. Validations of turbulent flow past a spinning cylinder with different velocity ratios were conducted and results showed reasonable agreement with available experimental measurement and numerical data.

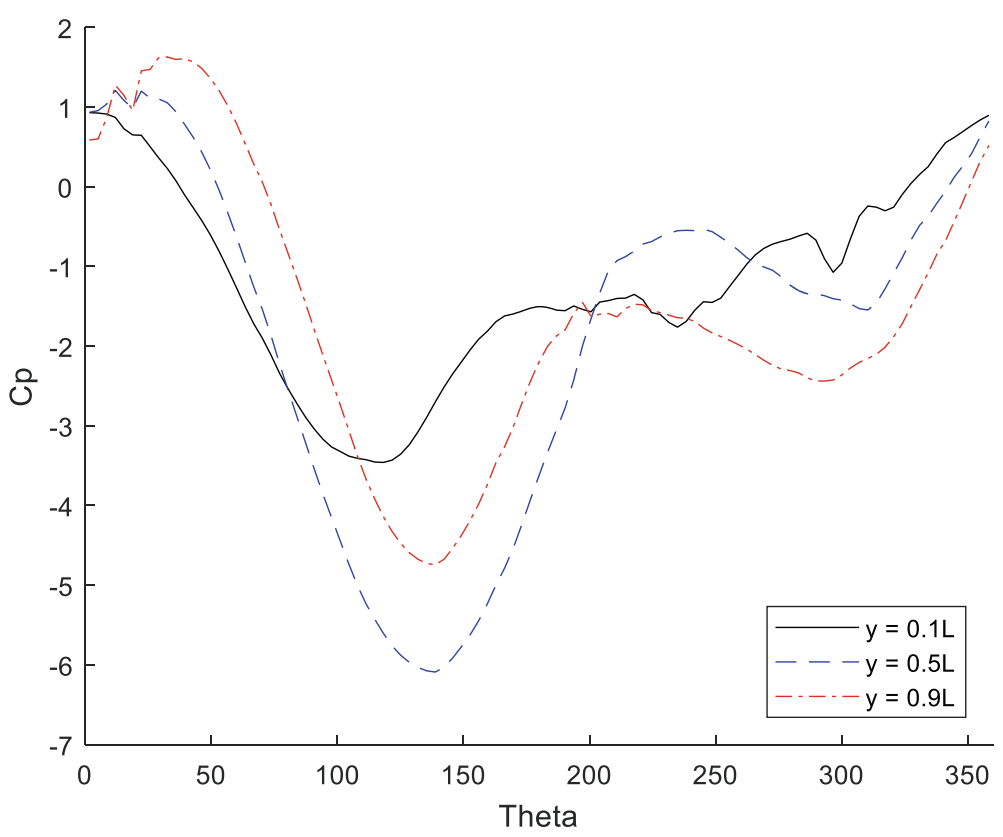

Fig. 11 Distribution of the pressure coefficient on the Magnus blade at different spanwise locations for $A R=3$ 

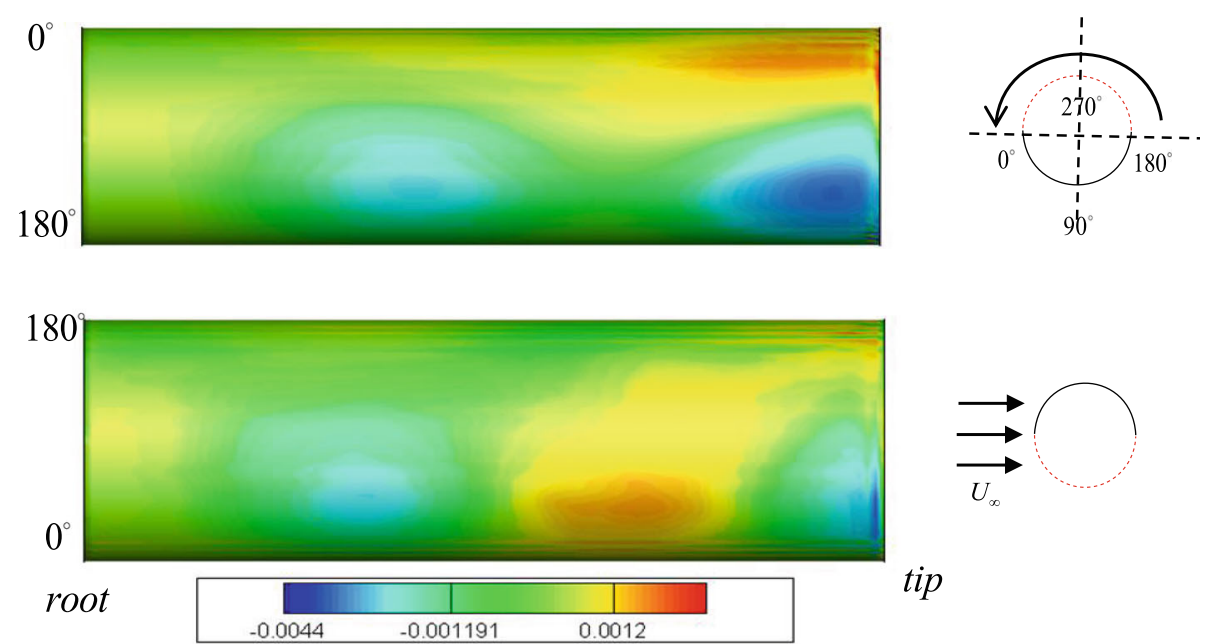

Fig. 12 Distribution of torque on the Magnus blade with $A R=3$. The projected surface is denoted by the black solid line

Further simulations of a spinning cylinder with differing aspect ratios were carried out to provide an insight on its influence on the Magnus effect. At the same Reynolds number of $1.0 \times 10^{5}$ and velocity ratio of 2.0 , the design with $A R=10$ showed a decrease in observed lift coefficient. The observed aspect ratio dependency was evidenced by the three-dimensionality of the near-wake behind the spinning cylinder.

The study was then extended to a single blade Magnus rotor, in which several conclusions were drawn. Regards to the case of solely spinning cylinder, the Magnus rotor with two different geometric designs and operating conditions both reported a significant jump in terms of mean drag coefficient. This is attributed to the increase of relative incidence angle between the blade and the in-coming flow. From the study of the distribution of pressure coefficients, the azimuthal angle of the base pressure is shifted

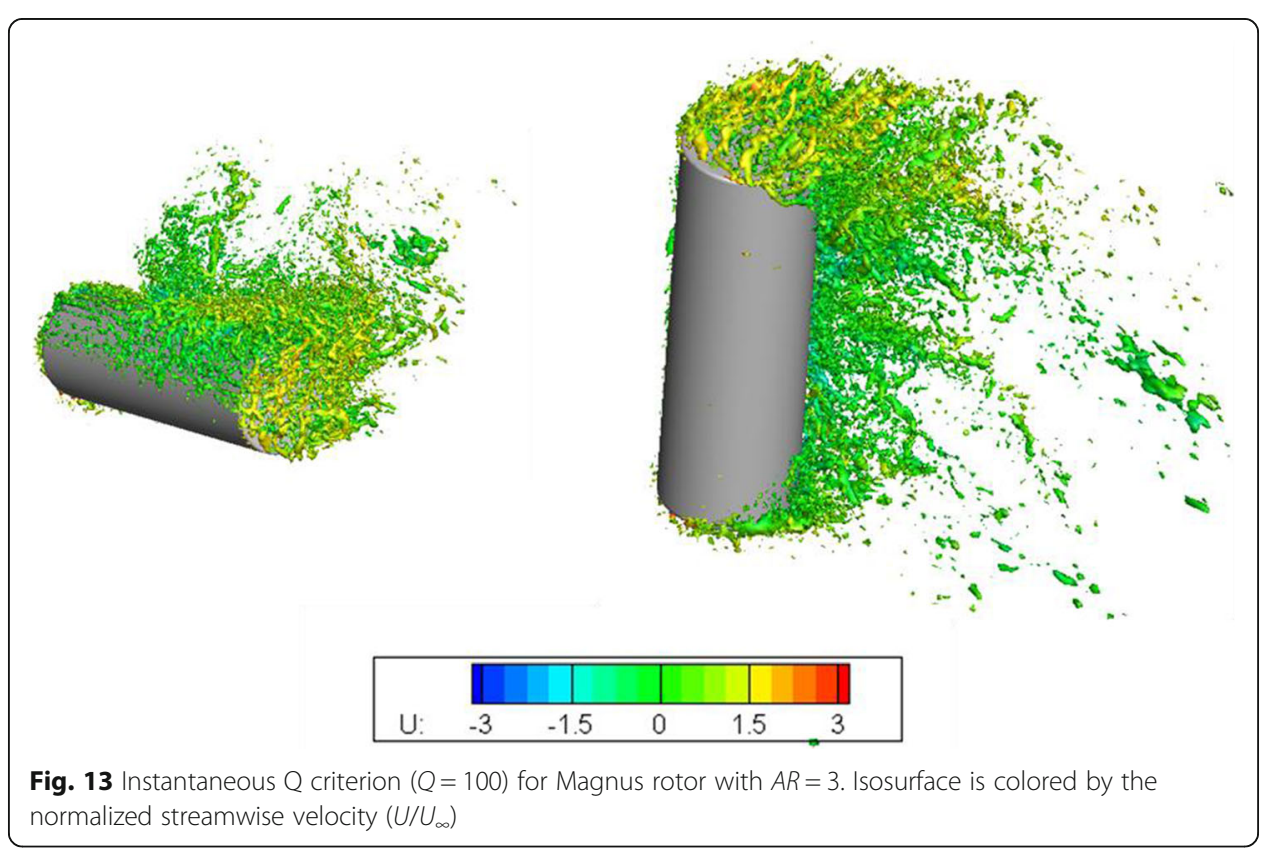


to a larger value, resulting in the loss of lift and increase of drag. An important remark on the fluctuations of the dynamic forcing is that the dominating frequency corresponds directly with the angular speed of the rotor's global motion of rotation, irrespective of the aspect ratio.

Between the two cases of Magnus rotor, the case with $A R=10$ showed a stronger Magnus effect with a relatively higher lift-to-drag ratio. Its superior performance in terms of power generation is clearly indicated by the dimensionless torque coefficient. During the rotation of the Magnus rotor, stable forces were expected at the root of the blade which are responsible for the major contribution of the total torque. For the case with $A R=3$, the combined effect of a weaker pressure difference between the pressure side and suction side of the cylinder and the shift of the base pressure was attributed to the reduction of the lift force. Clear differences for the turbulent structures surrounding the blade were also observed by the $\mathrm{Q}$ criterion. While both cases showed the vortices only shed from one side of the cylinder, a large structure was observed to be attached to the blade surface in the case with $A R=10$, where the surrounding flow has the highest streamwise velocity. For the case with $A R=3$, a stronger end effect was identified at both the root and tip of the blade.

Further studies should involve drag reduction measures, such as micro vortex generators, investigating the optimal aspect ratio and tip speed ratios for maximum power output as well as blade interaction. Moreover, the high efficiency of the novel circulating treadmill-like Magnus wind turbine blade introduced in the work by Kazemi et al. [13] is very promising, and thus it is worth carrying out three-dimensional LES simulations on the aerodynamic performance of the circulating airfoil, focusing on the control of undesirable dynamics issues.

\section{Nomenclatures}

$A R=L / D$ Aspect ratio.

$C_{\boldsymbol{a}}=\frac{f_{a}}{\frac{1}{2} \rho U_{\infty}^{2} \frac{\pi D^{2}}{4}}$ Axial Force Coefficient.

$C_{d}=\frac{f_{d}}{\frac{1}{2} \rho U_{\infty}^{2} D L}$ Drag Coefficient.

$C_{l}=\frac{f_{l}}{\frac{1}{2} \rho U_{\infty}^{2} D L}$ Lift coefficient.

$C_{p}=\frac{p}{\frac{1}{2} \rho U_{\infty}^{2}}$ Pressure coefficient.

$C_{\text {power }}=\frac{t_{s} \Omega}{\frac{1}{2} \rho U_{\infty}^{3} \frac{\pi D_{c}^{2}}{4}}$ Power Coefficient.

$C_{T}, C_{M T S}$ MTS parameters.

$C_{t_{s}}=\frac{t_{s}}{\frac{1}{2} \rho U_{\infty}^{2} D L^{2}}$ Dimensionless torque.

$D$ Cylinder diameter.

$D_{c}=L+L_{r}$ Magnus rotor diameter.

$F_{D}, F_{L}, F_{A}$ Drag, lift and axis forces in global coordinate system.

f Volume force.

$f_{d}, f_{l}, f_{a}$ Drag, lift and axis forces in local coordinate system.

$f_{s}$ Dominant vortex shedding frequency.

IBPs Immersed Boundary Points.

$k_{e s}$ Turbulent kinetic energy.

$L$ Cylinder length. 
$L_{r}$ Distance from the centre of rotation to cylinder root

$p$ Pressure.

$R e=U_{\infty} D / v$ Reynolds number.

$\bar{S}$ Strain-rate tensor.

$S t=\frac{f_{s} D}{U_{\infty}}$ Strouhal number.

$S t_{D_{c}}=\frac{f_{s} D_{c}}{\frac{1}{2} \Omega D_{c}}$ Strouhal number based on rotor diameter and rotation speed.

$T_{S}$ MTS time scale

$t_{s}$ Torque.

$T S R=\frac{D_{c} \Omega}{2 U_{\infty}}$ Tip Speed Ratio.

$\mathrm{U}$ Interpolated velocity.

$U_{\infty}$ Free stream velocity.

u Fluid velocity.

V Desired velocity.

$X, Y, Z$ Global coordinates.

$x, y, z$ Local coordinates.

$\alpha=\frac{D \omega}{2 U_{\infty}}$ Dimensionless velocity ratio.

$\Delta$ Averaged grid spacing.

$\theta$ Azimuthal angle.

$v$ Kinematic viscosity.

$v_{t}$ Turbulent viscosity.

$\rho$ Fluid density.

$\phi$ Rotation angle.

$\omega$ Cylinder spin speed.

$\Omega$ Rotor spin speed.

\section{Appendix}

7.1 Validation case - a stationary cylinder in turbulent flow

In this section, we investigated turbulent flow past a stationary circular cylinder at $R e=$ $1.4 \times 10^{5}$. The Reynolds number was selected because it is sub-critical for stationary cylinders and thus turbulence mainly develops in the wake instead of the boundary layer [33]. The dimensions of the computational box are $[-7.8 D, 17.8 D] \times[-12.8 D$, $12.8 D] \times[0,1.0 D]$ in $X, Y$ and $Z$ directions, respectively, while the cylinder, with a diameter of $D=1.0$ and length of $L=1.0$, is located at $(0.0,0.0,0.5)$. The computational domain is discretized on a stretched Cartesian mesh with a grid resolution of $256 \times$ $256 \times 48$ points. An embedded uniform sub-mesh with spacing of $D / 64$ around the cylinder is employed. The time step size is set to make the maximum Courant number approximately equal to 0.35 . The simulations were initially run for $80 \mathrm{D} / \mathrm{U}_{\infty}$ until the flow field became fully developed and were restarted for another $30 \mathrm{D} / U_{\infty}$ to gather the statistical moments. For flow past stationary cylinders, the sampling duration should cover at least 6 vortex shedding cycles.

Table A1 compares the mean drag force and the Strouhal number of turbulent flow past a stationary cylinder $(\alpha=0)$ with those of Karabelas [34] and Breuer [30], both of whom carried out large eddy simulations with the Smagrinsky SGS model on very fine body-conformal (BC) grids, and the experimental data from Cantwell \& Coles [35] who carried out a well-organized experiment. Note that, the same Reynolds number and 
cylinder's span-wise length $(L=D)$ were adopted in all three numerical simulations. It may be concluded that the present result and published numerical and experimental results are in good agreement. The Strouhal number converges to 0.2 for all three numerical simulations. The reported drag coefficient is close to the experimental data, but a difference is observed with other computations. Fig. A1 shows the time averaged streamlines and resolved turbulent kinetic energy in the near wake. From the velocity streamlines, two recirculation bubbles are present near the downstream of the cylinder and are nearly symmetric to the centreline at $Y=0$. In addition, two small counterrotating vortices are observed but closer to the surface of the cylinder. These small vortices were also identified in the cited study of Karabelas [34], who believes that they are due to the differences of the wall damping functions used in LES models. For stationary cylinder, Karabelas [34] stated that the major fraction of the energy comes from the vortex shedding rather than turbulent fluctuations. Therefore, the maximum kinetic energy should be expected in the near wake where the vortex shedding is dominant. Current results show the maximum kinetic energy around $x=1.2 D$, which broadly agrees with the value of $x=1.3 D$ reported by Karabelas [34].

Time averaged streamwise velocity along the centreline $(Y=0)$, the normal velocity and dimensionless shear stress at $X=D$ are plotted in Fig. A2 along with the available data from literature. From the streamwise velocity profile, present results agree well with respect to the cited LES results for the length of recirculation area but appear to under-predict the magnitude of the reversed flow. The same trend is also observed for velocity magnitude in the middle region but recovers quickly as it approaches the far wake. The normal velocity and shear stress distribution at $X=D$ show better agreement with the reference data for both magnitude and peak position, especially with the computed results from Breuer [30].

\subsection{Validation case - a spinning cylinder in turbulent flow}

In this section, we investigated turbulent flow past a spinning circular cylinder at $R e=$ $1.4 \times 10^{5}$. From the studies of Karabelas [34] and Karabelas et al. [36], it is reported that a minimum velocity ratio of 2.0 is required to suppress vortex shedding, hence is of particular interest to maintain the desired forces on the cylinder to ensure the rotor movement. For flow past the spinning cylinder, the rotating fluid driven by the motion of the cylinder is superimposed on the free-stream flow, hence will lead to a global increase of the fluid's velocity. In this case, the mesh resolution used in the previous case may not be adequate to predict the correct aerodynamic forces. To investigate the optimal mesh/IBPs resolution for this study, a grid dependency was carried out with three different spacings in the sub-mesh region around the cylinder, i.e., $D / 32, D / 64$ and $D /$ 128. To keep the computational effort a reasonable level, the same number of overall grids as the previous for the case of stationary cylinder was used and the computational domain was kept the same by adjusting the stretching ratios of the outer grids. In each case, the number of IBPs was adjusted to meet the requirement that there is at least one point inside each Cartesian grid. For example, for RUN-32, where the uniform grid spacing is set as $D / 32,128$ IBPs were employed around the perimeter of the cylinder in the horizontal plane. For all simulations, 48 layers of IBPs were uniformly distributed along the spanwise $(Z)$ direction. The time step size was set as $0.004 D / U_{\infty}, 0.002 \mathrm{D} /$ 
$U_{\infty}, 0.001 \mathrm{D} / U_{\infty}$ to make the max Courant number approximately equal to 0.35 for all cases.

The statistical features from all three studies are presented in Table A2 along with published numerical and experimental results. Clearly, these results are quite diverse. The smallest drag coefficient of 0.13 is observed in the numerical results of Karabelas [34] and the largest one of $0.7 \sim 1.2$ is reported by Borg [1] who compiled many experimental results of flow past a spinning cylinder with different Reynolds numbers, end plate's diameter, surface roughness and aspect ratios. Among the present study, all three simulations seem to over-predict the drag coefficient as compared to the computation results of Karabelas [34] and are below the experimental results. For the lift force, both RUN-64 and RUN-128 predicted lower coefficient than the published LES data while RUN-32 showed a much higher value.

The time averaged pressure coefficient along the cylinder surface is shown in Fig. A3 with the origin located at the front of the cylinder facing the inflow. For all three cases, the pressure coefficients along the upper half of the cylinder match well with each other and agree with the trend of the reference data. The minimum coefficient exceeds -6 for all cases, which clearly indicates a reduction of static pressure due to the spinning of the cylinder. Quantitively, the more rapid drop of the pressure value in the cited LES data can be used to explain the previously observed difference in the lift coefficient. On the lower half, RUN-64 shows an evident dip in $C_{p}$ before the stagnation point, where the pressure coefficient reaches its peak value. At the back of the cylinder $\left(150^{\circ}<\theta<200^{\circ}\right)$, the absolute value of static pressure is lower in the current studies as compared to the LES data, resulting in a higher drag coefficient. Closer to the azimuthal origin, the three curves coincide with each other and the computed data of Karabelas [34].

The streamlines plotted using the time-averaged velocity, are also presented for comparison in Fig. A4. Like the results of Karabelas [34], only one distinct recirculation bubble remains in both RUN-32 and RUN-64 while the other one collapses. In RUN128 , the upper recirculation bubble is still present but is of much smaller size compared to the lower one. In the later study of Karabelas et al. [36], in which a RANS code was applied, a similar pattern was observed for $\alpha=2.0$ at a higher Reynolds number ( $R e=$ $5.0 \times 10^{5}$ ). An explanation is that the flow is at the transitional stage where the vortex shedding is damped but not completely diminished. From the total resolved kinetic energy and shear stress plots for RUN-64, see Fig. A5, high values of kinetic energy, though much smaller compared with the stationary cylinder, can still be seen in the near wake, indicating the presence of the periodic oscillation induced by the vortex shedding. Similarly, the maximum value of Reynolds stress lies on the downwind side of the cylinder, influenced by the high momentum of fluid coming from the upper side of the cylinder. Around the cylinder, positive values of stress are observed near the front stagnation point and the minimum stress is seen on the lower surface of the cylinder, of which the absolute value is still smaller than the near wake, implying the turbulent fluctuations have not yet become dominant. The previously observed dip in $C_{p}$ value for RUN-64 can be explained by the elongated recirculation bubble at the downwind side of the cylinder, which covers a wider azimuthal area $\left(200^{\circ}<\theta<300^{\circ}\right)$ and is closer to the cylinder's surface.

From the grid dependency study, all cases show the spinning of the cylinder has greatly altered the flow field as well as damped the vortex shedding process. Results 
with the coarsest grid resolution tend to overpredict the lift coefficients and offset the stagnation point. On the other hand, simulations with refined grid spacing of $D / 64$ and $D / 128$ are in reasonable agreement, although the largest differences are observed for the drag coefficient, which stem from the pressure distribution along the cylinder circumference. In general, the overall behaviour of the pressure coefficient distribution is in quite good agreement with experimental and numerical results from the literature. Given these results, the refined grid is necessary in the uniform sub-mesh region to capture the dynamic loadings. Thus, the meshes in the following numerical simulations, unless stated otherwise, were refined to allow at least 64 grid points along the diameter of the cylinder.

\section{Supplementary Information}

The online version contains supplementary material available at https://doi.org/10.1186/s42774-021-00068-9.

Additional file 1: Table A1. Comparison of drag coefficient, lift coefficient and Strouhal number for flow past a stationary circular cylinder at $R e=1.4 \times 10^{5}$ and $a=0$.

Additional file 2: Table A2. Comparison of drag coefficient, lift coefficient and stagnation point for flow past a spinning circular cylinder at $\operatorname{Re}=1.4 \times 10^{5}$ and $a=2$. Results from Karabelas et al. [36] is for $\operatorname{Re}=5 \times 10^{5}$ and $a=2$.

Additional file 3: Figure A1. Streamlines of flow and contours of the total resolved kinetic energy in the near wake for flow past a stationary circular cylinder with $R e=1.4 \times 10^{5}$ and $a=0$.

Additional file 4: Figure A2. Time averaged streamwise velocity along the centreline and time averaged normal velocity and dimensionless shear stress at $X=D$ for flow past a stationary circular cylinder with $\operatorname{Re}=1.4 \times 10^{5}$ and $a=0$.

Additional file 5: Figure A3. Distribution of pressure coefficient along the cylinder's surface for different azimuthal angles with $R e=1.4 \times 10^{5}$ and $a=2.0$.

Additional file 6: Figure A4: Contours of dimensionless pressure coefficient and streamlines of flow past a spinning cylinder with $R e=1.4 \times 10^{5}$ and $a=2.0$ : (a) RUN-32; (b) RUN-64; (c) RUN-128; (d) Streamlines of Karabelas [34].

Additional file 7: Figure A5. Contours of the total resolved kinetic energy and shear stress plotted in the near wake for flow past a spinning circular cylinder with $R e=1.4 \times 10^{5}$ and $a=2.0$.

\section{Acknowledgements}

The authors kindly acknowledge Grant Applied Sciences and Innovate UK for their support, and EPSRC turbulence consortium for providing national computing time under grant EP/L000261/1.

\section{Authors' contributions}

The contribution of the authors to this work is equivalent. All authors read and approved the final manuscript.

\section{Funding}

Not applicable.

Availability of data and materials

All data and materials are available from the authors of this paper.

\section{Declaration}

Competing interests

The authors declare that they have no competing interests.

\section{Author details}

${ }^{1}$ School of Engineering and Materials Science, Queen Mary University of London, Mile End Road, London E1 4NS, UK. ${ }^{2}$ Siemens Ltd., China, Technology, Beijing 100102, China. ${ }^{3}$ State Key Laboratory of Hydraulic Engineering Simulation and Safety, Tianjin University, Tianjin 300072, China. ${ }^{4}$ Grant Applied Sciences, 3rd Floor, 3 Fitzhardinge Street, London W1H 6E, UK

Received: 23 February 2021 Accepted: 10 May 2021

Published online: 12 July 2021

\section{References}

1. Borg J (1986) Magnus effect: an overview of its past and future practical applications. The Borg/Luther Group, Naval Sea Systems Command Contract (00024)

2. Seifert J (2012) A review of the Magnus effect in aeronautics. Prog Aerosp Sci 55:17-45. https:/doi.org/10.1016/j.paerosci.2012.07.001

3. Tokumaru P, Dimotakis P (1993) The lift of a cylinder executing rotary motions in a uniform flow. J Fluid Mech 255(1):110. https://doi.org/10.1017/S0022112093002368

4. Mittal S, Kumar B (2003) Flow past a rotating cylinder. J Fluid Mech 476(4):303-334. https://doi.org/10.1017/S0022112002002938

5. Thom A (1934) Effect of discs on the air forces on a rotating cylinder. HM Stationery Office, London 
6. Badalamenti C, Prince S (2008) Effects of endplates on a rotating cylinder in crossflow. AIAA 2008-7063. 26th AIAA Applied Aerodynamics Conference

7. Chauvin A, Benghrib D (1989) Drag and lift coefficients evolution of a Savonius rotor. Exp Fluids 8(1):118-120. https:// doi.org/10.1007/BF00203076

8. Iversen J (1969) The Magnus rotor as an aerodynamic decelerator. AIAA 1968-962. 2nd Aerodynamic Deceleration Systems Conference

9. Saha U, Rajkumar MJ (2006) On the performance analysis of Savonius rotor with twisted blades. Renew Energy 31(11): 1776-1788. https://doi.org/10.1016/j.renene.2005.08.030

10. Akira I, Kawashima S, Nishizawa Y, Ushiyama I, Komatinovic N (2007) A study on Savonius type Magnus wind turbine. Paper presented at the European Wind Energy Conference \& Exhibition 2007, Milan

11. Sedaghat A (2014) Magnus type wind turbines: prospectus and challenges in design and modelling. Renew Energy 62: 619-628. https://doi.org/10.1016/j.renene.2013.08.029

12. Richmond-Navarro G, Calderón-Muñoz WR, LeBoeuf R, Castillo P (2017) A Magnus wind turbine power model based on direct solutions using the blade element momentum theory and symbolic regression. IEEE Transactions on Sustainable Energy 8(1):425-430. https://doi.org/10.1109/TSTE.2016.2604082

13. Kazemi SA, Nili-Ahmadabadi M, Sedaghat A, Saghafian M (2016) Aerodynamic performance of a circulating airfoil section for Magnus systems via numerical simulation and flow visualization. Energy 104:1-15. https://doi.org/10.1016/.jenergy.2016.03.115

14. McCormick BW (1967) Aerodynamics of V/STOL flight. Courier Corporation, North Chelmsford

15. Ji C, Munjiza A, Williams J (2012) A novel iterative direct-forcing immersed boundary method and its finite volume applications. J Comput Phys 231(4):1797-1821. https://doi.org/10.1016/j.jcp.2011.11.010

16. Thomas T, Williams J (1997) Development of a parallel code to simulate skewed flow over a bluff body. J Wind Eng Ind Aerodyn 67:155-167. https://doi.org/10.1016/S0167-6105(97)00070-6

17. Ji C, Munjiza A, Avital E, Ma J, Williams J (2013) Direct numerical simulation of sediment entrainment in turbulent channel flow. Phys Fluids 25(5):056601. https://doi.org/10.1063/1.4807075

18. Ji C, Munjiza A, Avital E, Xu D, Williams J (2014) Saltation of particles in turbulent channel flow. Phys Rev E 89(5):052202. https://doi.org/10.1103/PhysRevE.89.052202

19. Xu D, Kaliviotis E, Munjiza A, Avital E, Ji C, Williams J (2013) Large scale simulation of red blood cell aggregation in shear flows. J Biomech 46(11):1810-1817. https://doi.org/10.1016/.jbiomech.2013.05.010

20. Xu D, Ji C, Munjiza A, Kaliviotis E, Avital E, Willams J (2019) Study on the packed volume-to-void ratio of idealized human red blood cells using a finite-discrete element method. Applied Mathematics Mechanics (English Edition) 40(5): 737-750. https://doi.org/10.1007/s10483-019-2473-6

21. Bai X, Avital E, Munjiza A, Williams J (2014) Numerical simulation of a marine current turbine in free surface flow. Renew Energy 63:715-723. https://doi.org/10.1016/j.renene.2013.09.042

22. Chen W, Ji C, Xu D, Srinil N (2019) Wake patterns of freely vibrating side-by-side circular cylinders in laminar flows. J Fluids Structures 89:82-95. https://doi.org/10.1016/j.jfluidstructs.2019.02.013

23. Chen W, Ji C, Alam MM, Williams J, Xu D (2020) Numerical simulations of flow past three circular cylinders in equilateraltriangular arrangements. J Fluid Mech 891:A14. https://doi.org/10.1017/jfm.2020.124

24. Ji C, Cui Y, Xu D, Yang X, Srinil N (2019) Vortex-induced vibrations of dual-step cylinders with different diameter ratios in laminar flows. Phys Fluids 31(7):073602. https://doi.org/10.1063/1.5097730

25. Yan Y, Ji C, Srinil N (2020) Three-dimensional flip-flopping flow around a pair of dual-stepped circular cylinders in a sideby-side arrangement. Phys Fluids 32(12):123608. https://doi.org/10.1063/5.0031062

26. Zhang Z, Ji C, Chen W, Hua Y, Srinil N (2021a) Influence of boundary layer thickness and gap ratios on threedimensional flow characteristics around a circular cylinder in proximity to a bottom plane. Ocean Eng 226:108858. https://doi.org/10.1016/j.oceaneng.2021.108858

27. Zhang Z, Ji C, Xu D (2021 b) Temporal and spatial evolution of vortex shedding for flow around a cylinder close to a wall. Ocean Eng 228:108964. https://doi.org/10.1016/j.oceaneng.2021.108964

28. Singh K, Avital E, Williams J, Ji C, Bai X, Munjiza A (2017) On parallel pre-conditioners for pressure Poisson equation in LES of complex geometry flows. Int J Numer Methods Fluids 83(5):446-464. https://doi.org/10.1002/fld.4277

29. Inagaki M, Kondoh T, Nagano Y (2005) A mixed-time-scale SGS model with fixed model-parameters for practical LES. J Fluids Eng 127(1):1-13. https://doi.org/10.1115/1.1852479

30. Breuer M (2000) A challenging test case for large eddy simulation: high Reynolds number circular cylinder flow. Int J Heat Fluid Flow 21(5):648-654. https://doi.org/10.1016/S0142-727X(00)00056-4

31. Craft T, Johnson N, Launder B (2014) Back to the future? A re-examination of the aerodynamics of Flettner-Thom rotors for maritime propulsion. Flow Turbulence Combustion 92(1-2):413-427. https://doi.org/10.1007/s10494-013-9486-4

32. Rolfo S, Revell A (2015) Effect of span-wise resolution for LES of flow over a rotating cylinder at high Reynolds number. In: Direct and Large-Eddy Simulation IX. springer, pp 479-486

33. Yu G, Avital E, Williams J (2008) Large eddy simulation of flow past free surface piercing circular cylinders. J Fluids Eng 130(10):101304. https://doi.org/10.1115/1.2969462

34. Karabelas S (2010) Large eddy simulation of high-Reynolds number flow past a rotating cylinder. Int J Heat Fluid Flow 31(4):518-527. https://doi.org/10.1016/j.jiheatfluidflow.2010.02.010

35. Cantwell B, Coles D (1983) An experimental study of entrainment and transport in the turbulent near wake of a circular cylinder. J Fluid Mech 136(1):321-374. https://doi.org/10.1017/S0022112083002189

36. Karabelas S, Koumroglou B, Argyropoulos C, Markatos N (2012) High Reynolds number turbulent flow past a rotating cylinder. Appl Math Model 36(1):379-398. https://doi.org/10.1016/j.apm.2011.07.032

\section{Publisher's Note}

Springer Nature remains neutral with regard to jurisdictional claims in published maps and institutional affiliations. 